\title{
Solar Oscillations and Convection: II. Excitation of Radial Oscillations
}

\author{
R. F. Stein \\ Dept. of Physics and Astronomy, Michigan State University, East Lansing, MI 48823, U.S.A. \\ stein@pa.msu.edu \\ and
}

Å. Nordlund

Theoretical Astrophysics Center and Astronomical Observatory / NBIfAFG, Juliane Maries Vej 30, Dk-2100 Copenhagen Ø, Denmark

aake@astro.ku.dk

\begin{abstract}
Solar $p$-mode oscillations are excited by the work of stochastic, non-adiabatic, pressure fluctuations on the compressive modes. We evaluate the expression for the radial mode excitation rate derived by Nordlund \& Stein (2000) using numerical simulations of near surface solar convection. We first apply this expression to the three radial modes of the simulation and obtain good agreement between the predicted excitation rate and the actual mode damping rates as determined from their energies and the widths of their resolved spectral profiles. These radial simulation modes are essentially the same as the solar modes at the resonant frequencies, where the solar modes have a node at the depth of the bottom of the simulation domain. We then apply this expression for the mode excitation rate to the solar modes and obtain excellent agreement with the low $\ell$ damping rates determined from GOLF data. Excitation occurs close to the surface, mainly in the intergranular lanes and near the boundaries of granules (where turbulence and radiative cooling are large). The non-adiabatic pressure fluctuations near the surface are produced by small instantaneous local imbalances between the divergence of the radiative and convective fluxes near the solar surface. Below the surface, the non-adiabatic pressure fluctuations are produced primarily by turbulent pressure fluctuations (Reynolds stresses). The frequency dependence of the mode excitation is due to effects of the mode structure and the pressure fluctuation spectrum. Excitation is small at low frequencies due to mode properties - the mode compression decreases and the mode mass increases at low frequency. Excitation is small at high frequencies due to the pressure fluctuation spectrum - pressure fluctuations become small at high frequencies because they are due to convection which is a long time scale phenomena compared to the dominant $p$-mode periods.
\end{abstract}

Subject headings: sun:oscillations- sun:p-modes- sun:convection- sun:numerical simulation

\section{Introduction}

Two ideas for the source of $p$-mode excitation have been pursued - overstability (as in pulsating stars) and turbulent Reynolds stresses (as in jet noise) (Biermann 1948; Schwarzschild
1948; Lighthill 1952; Stein 1967, 1968; Crighton 1975; Ando \& Osaki 1977; Goldreich \& Keeley 1977; Goldreich \& Kumar 1990; Balmforth 1992; Musielak et al. 1994). We show here that it is the $P d V$ work of stochastic, non-adiabatic pressure fluctuations which is the primary mode excitation 
mechanism (Stein \& Nordlund 1991; Bogdan et al. 1993; Goldreich et al. 1994). Near the surface, the non-adiabatic gas pressure (i.e. entropy) fluctuations dominate. They are produced by radiative cooling at the solar surface which is not locally and instantaneously exactly balanced by convective heat deposition. Below the surface, non-adiabatic turbulent pressure (Reynolds stress) fluctuations dominate. They are produced by the turbulent convective motions.

In a previous paper (Nordlund \& Stein 2000) we derived an exact expression for the stochastic excitation rate of the radial solar $p$-modes by the $P d V$ work of non-adiabatic gas and turbulent pressure fluctuations on the mode compression. We now use realistic numerical simulations of near surface solar convection (depth about $2.5 \mathrm{Mm}$ ) to evaluate this expression (Stein \& Nordlund 1998). Because the largest entropy and pressure fluctuations occur near the solar surface and because modes with frequencies in the $3-4 \mathrm{mHz}$ range, where the excitation rate is largest, are confined near the solar surface, these near surface simulations include the primary excitation and damping processes.

\section{Mode Excitation: Formalism}

The rate of energy input to the modes can be calculated starting with the kinetic energy equation for the modes (Nordlund \& Stein 2000). Neglecting the viscous stresses,

$$
\begin{aligned}
\rho \frac{D}{D t}\left(\frac{1}{2} u_{z}^{2}\right) & =-\frac{\partial}{\partial z}\left[u_{z}\left(P_{g}+P_{t}\right)\right]+\rho u_{z} g \\
& +\left(P_{g}+P_{t}\right) \frac{\partial u_{z}}{\partial z} .
\end{aligned}
$$

After integrating this equation over depth, the flux divergence term only gives contributions from the end points and is negligible. The buoyancy term is small because mass is conserved so there is no net mass flux. The last term is the $P d V$ work,

$$
W=\int d t \int d z \delta P \frac{\partial \dot{\xi}}{\partial z}
$$

There are several contributions to this work. The displacement, $\xi$, has contributions from the modes as well as the random convective motions. The pressure, $\delta P$, has coherent contributions from the modes and incoherent contributions from the random convective motions. Both coherent and incoherent contributions can be further divided into adiabatic and non-adiabatic terms. The dominant driving comes from the interaction of the nonadiabatic, incoherent pressure fluctuations,

$$
\delta P^{\text {nad }}=\left(\delta \ln P-\Gamma_{1} \delta \ln \rho\right) P,
$$

with the coherent mode displacement,

$$
\frac{D \ln \rho}{D t}=-\frac{\partial \dot{\xi}}{\partial z}
$$

This is a stochastic process, so the pressure fluctuations occur with random phases with respect to the modes. Therefore one must average over all possible relative phases between them. The resulting rate of energy input to the modes is (Nordlund \& Stein 2000)

$$
\frac{\Delta\left\langle E_{\omega}\right\rangle}{\Delta t}=\frac{\omega^{2}\left|\int_{r} d r \delta P_{\omega}^{*} \frac{\partial \xi_{\omega}}{\partial z}\right|^{2}}{8 \Delta \nu E_{\omega}} .
$$

Here, $\delta P_{\omega}$ is the time Fourier transform of the nonadiabatic total pressure. $\Delta \nu=1 /$ (total time interval) is the frequency resolution with which $\delta P_{\omega}$ is computed. $\xi_{\omega}$ is the mode displacement eigenfunction, which is typically chosen to be real for an adiabatic mode. In that case, taking the complex conjugate of the pressure is not necessary, but we retain it for generality. The mode energy is

$$
E_{\omega}=\frac{1}{2} \omega^{2} \int_{r} d r \rho \xi_{\omega}^{2}\left(\frac{r}{R}\right)^{2}=M_{\omega} V_{\omega}^{2}(R)
$$

Here $M_{\omega}$ is the mode mass and $V_{\omega}(R)$ is the mode velocity amplitude at the surface. Eqn 5 is similar to the expression of Goldreich et al. (1994) (eqn. 26), but involves no approximations. Having the numerical simulation data, we can evaluate this expression exactly without having to make approximations in order to evaluate it analytically 1

\section{Simulations}

We simulate a small portion of the solar photosphere and the upper layers of the convection zone, a region extending $6 \times 6 \mathrm{Mm}$ horizontally and from the temperature minimum at $-0.5 \mathrm{Mm}$ down to $2.59 \mathrm{Mm}$ below the visible surface. We

\footnotetext{
${ }^{1} \mathrm{~A}$ detailed description of how eqn 5 is evaluated is given in the appendix. Its validity is tested by applying it to the three radial modes of the simulation domain (sec. 4).
} 
solve the equations of mass, momentum and energy conservation in the form:

$$
\begin{aligned}
\frac{\partial \ln \rho}{\partial t}= & -\mathbf{u} \cdot \nabla \ln \rho-\nabla \cdot \mathbf{u}, \\
\frac{\partial \mathbf{u}}{\partial t}= & -\mathbf{u} \cdot \nabla \mathbf{u}+\mathbf{g}-\frac{P}{\rho} \nabla \ln P+\frac{1}{\rho} \nabla \cdot \underline{\underline{\sigma}}(8) \\
\frac{\partial e}{\partial t}= & -\mathbf{u} \cdot \nabla e-\frac{P}{\rho} \nabla \cdot \mathbf{u} \\
& +Q_{\text {rad }}+Q_{\text {visc }},
\end{aligned}
$$

where $\underline{\underline{\sigma}}$ is the viscous stress tensor, $Q_{\text {rad }}$ is the radiative heating and $Q_{v i s c}$ is the viscous dissipation.

We use a non-staggered grid of either $125^{2}$ cells horizontally $\times 82$ cells vertically or $63^{3}$ cells. The spatial derivatives are calculated using third order splines and the time advance is a third order leapfrog scheme (Hyman 1979; Nordlund \& Stein 1990). The code is stabilized by a hyperviscosity which removes short wavelength noise without damping the longer wavelengths.

A large fraction of the internal energy is in the form of ionization energy near the solar surface, so we use a realistic equation of state (including the effects of ionization and excitation of hydrogen and other abundant elements and the formation and ionization of $\mathrm{H}_{2}$ molecules). The pressure is found by interpolation in a table of $P(\ln \rho, e)$ and its derivatives which is calculated with the Uppsala stellar atmosphere package (Gustafsson et al. 1975).

Radiative energy exchange is critical in determining the structure of the upper convection zone. Near the surface of the sun, the energy flow changes from almost exclusively convective below the surface to radiative above the surface. The interaction between convection and radiation near the solar surface determines what we observe and produces the entropy fluctuations that lead to the buoyancy work which drives the convection and that give rise to the non-adiabatic pressure fluctuations which excite the p-mode oscillations. Hence, the interaction between convection and radiation is crucial for both the diagnostics and the dynamics of convection. Since the top of the convection zone occurs near the level where the continuum optical depth is one, neither the optically thin nor the diffusion approximations give reason- able results. We therefore include 3D, LTE, nongray radiation transfer in our model.

We simulate only a small region near the solar surface and must therefore impose boundary conditions inside the convectively unstable region. What happens outside our computational domain in principle influences the convective motions inside. However, at the top boundary, the density is very low relative to the rest of the volume and hence whatever happens there has very little influence on the convective motions. At the bottom, the incoming fluid is to a very good approximation isentropic and featureless, and hence carries little information. The unknown influence of external regions should therefore be small. This assertion is indeed confirmed by experiments with boundaries located at different depths.

The horizontal directions are taken to be periodic. In the vertical direction, we have a transmitting boundary at the temperature minimum (Nordlund \& Stein 1990). This is achieved by a larger than normal zone at the top boundary. Across this zone we make the vertical derivative of the density hydrostatic, set the vertical derivative of the velocity zero and hold the internal energy at the top fiducial layer constant in time and space. At the bottom of the computational domain, outgoing fluid goes out with whatever properties it has. For incoming fluid, we adjust the pressure such that the net mass flux through the bottom boundary vanishes. (This ensures that there is no boundary work done on vertical oscillation modes.) The pressure on the bottom boundary thus varies in time but is uniform over the horizontal plane. We damp fluctuations of the horizontal and vertical velocity of the incoming fluid, using a long time constant. Finally, we adjust the density and energy of the incoming fluid, at constant pressure, to fix its entropy (in both space and time).

The ability to do a direct numerical simulation with the wide range of length scales matching the dimensionless parameters - Reynolds number, Rayleigh number and Prandtl number - of the solar convection zone is beyond the speed and memory capabilities of current computers. Thus, our simulations are of the type called "Large Eddy Simulations". It is, however, possible to resolve the surface thermal boundary layer of the convection zone and this we have done. Indeed, this is re- 
quired to achieve results that agree quantitatively with solar observations (Stein \& Nordlund 2000).

The picture of convection that has emerged from the simulations is the following: Convection is driven by radiative cooling in the thin thermal boundary layer at the solar surface. It consists of cool, low entropy, filamentary, turbulent, downdrafts that plunge through a warm, entropy neutral, smooth, diverging, laminar upflow. Upflows must diverge as they ascend into lower density layers in order to conserve mass. This divergence smooths out any variations in their properties that might arise. Only a small fraction of the fluid at depth reaches the surface to be cooled and form the cores of the downdrafts. Most fluid turns over within a scale height and is entrained by the downdrafts. These low entropy downflows are the site of most of the buoyancy work that drives the convection. (See Stein \& Nordlund (1998) for more details.)

We have made numerous comparisons between the predictions of the simulations and solar observations:

- The depth of the convection zone depends on the opacities that determine the temperature versus pressure (and hence entropy) stratification of the surface layers, the spectral line blocking, the convective efficiency of the superadiabatic layers immediately below the surface that determines the transition to the asymptotic adiabat, and the equation of state that determines the further run of temperature versus pressure through the convection zone. Excellent agreement is obtained between the depth of the convection zone predicted by our numerical simulations and that inferred from helioseismology (Rosenthal et al. 1999).

- The cavity for high frequency modes is enlarged by turbulent pressure support and 3D non-linear opacity effects which increase the average temperature required to produce a given effective temperature in an inhomogeneous compared to a homogeneous atmosphere. The $p$-mode eigenfrequencies calculated from the mean simulation atmosphere are significantly closer to the observed mode frequencies than those for standard spherically symmetric, mixing length models (Rosenthal et al. 1998, 1999).

- The simulation granulation size spectrum and the distribution of emergent intensities, when smoothed by the point spread function appropriate for the Swedish Vacuum Solar Telescope on La Palma (which produces the best solar images available today), closely match the observations (Stein \& Nordlund 1998).

- The width of photospheric iron lines (whose thermal speed is small) is a signature of the convective velocities. The net Doppler blue shift and asymmetry of spectral lines is a signature of the correlation between velocity and temperature fluctuations. Both these signatures agree closely with observations (Asplund et al. 2000).

This gives us confidence that the simulations are properly modeling the crucial properties of near surface solar convection.

\section{Mode Excitation: Simulation}

Three radial modes exist in our simulation and we first apply eqn (5) to these modes and compare the rate of work on the modes it predicts with the actual excitation rate $d E_{\omega} / d t=\Gamma E_{\omega}$ determined from the mode's energies and widths in the simulation. The modes can be clearly seen in the spectrum of the horizontally averaged, depth integrated kinetic energy (fig 1). Their properties are given in table 1 . The lowest mode (with no zero crossings inside the computational domain) has a frequency of $2.57 \mathrm{mHz}$ and a FWHM of 19 $\mu \mathrm{Hz}$. Hence a simulation significantly longer than $14.5 \mathrm{hrs}$ is required to resolve this mode. We use a simulation of 43 hours, with a spatial resolution of $63^{3}$. Snapshots were saved at $30 \mathrm{~s}$ intervals.

Table 1: Mode Properties

\begin{tabular}{|c|c|c|c|c|c|}
\hline $\begin{array}{c}\nu \\
(\mathrm{mHz})\end{array}$ & $\begin{array}{c}\Delta \nu \\
(\mu \mathrm{Hz})\end{array}$ & $\begin{array}{c}E_{\omega} \\
(\mathrm{ergs})\end{array}$ & $\mathrm{Q}$ & $\begin{array}{c}\Gamma \\
\left(\mathrm{s}^{-1}\right)\end{array}$ & $\begin{array}{c}\mathrm{dE} / \mathrm{dt} \\
\left(\mathrm{erg} \mathrm{s}^{-1}\right)\end{array}$ \\
\hline 2.57 & 19.0 & $3.4 \times 10^{26}$ & 135 & $1.2 \times 10^{-4}$ & $4.3 \times 10^{22}$ \\
\hline 3.88 & 133 & $2.6 \times 10^{25}$ & 29 & $8.4 \times 10^{-4}$ & $2.2 \times 10^{22}$ \\
\hline 5.58 & 438 & $2.2 \times 10^{24}$ & 13 & $2.7 \times 10^{-3}$ & $6.1 \times 10^{21}$ \\
\hline
\end{tabular}




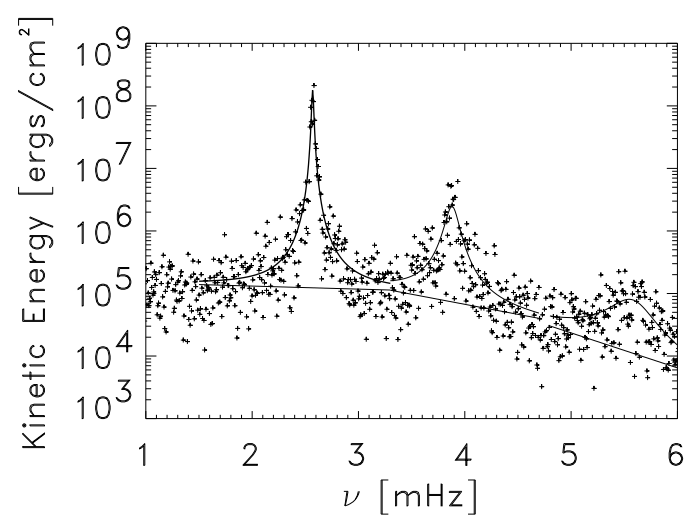

Fig. 1.- Kinetic energy, horizontally averaged and integrated over depth. Three radial modes are clearly visible. Least squares, Lorentzian, fits to the modes and linear fits to the background noise are superimposed.

The eigenfunctions of the velocity are calculated by taking the time Fourier transform of the velocity. To get the real eigenmode, the transform of the velocity at the frequency of the mode is divided by its most common phase among all depths. To reduce the noise, we average the result over a frequency band, approximately equal to the FWHM of the mode, centered on the mode. The eigenfunctions are essentially the same as the solar model modes of Christensen-Dalsgaard using his spherically symmetric model S (ChristensenDalsgaard et al. 1996) at the mode frequencies (fig 2), when the modes are normalized by the square root of the mode energy, or which is equivalent by their amplitude at the surface. Hence, we choose to use the solar model eigenfunctions, because they are much denser (35 radial modes below the acoustic cutoff frequency instead of three) and because they are slightly smoother. Another way of looking at the modes is via their kinetic energy. Figure 3 is an image of the logarithm of the kinetic energy as a function of depth and frequency. The three modes of the computational domain are clearly seen. Notice also that there is a continuous pattern of nodes and antinodes, with the nodes descending in depth as the frequency increases and new nodes starting at the surface. This pattern extends even into the propagating region above the acoustic cutoff frequency of about $5.3 \mathrm{mHz}$.

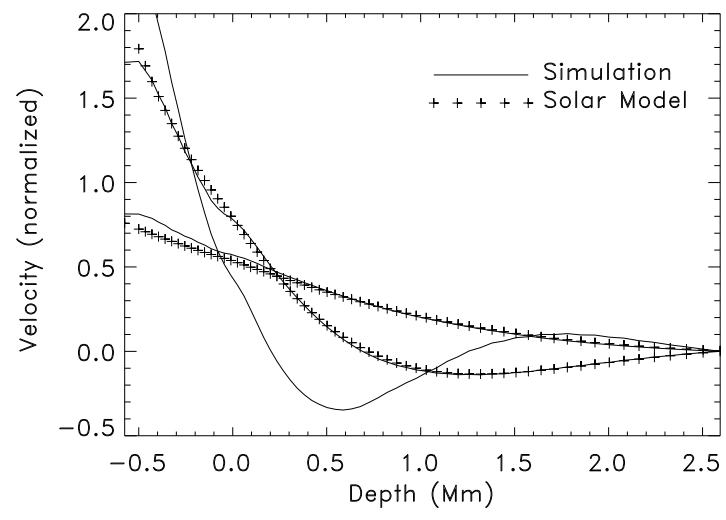

Fig. 2.- Velocity eigenmodes of the simulation compared with those of a solar model (model S of Christensen-Dalsgaard et al. 1996). The modes are normalize by the square root of their energy (eqn 6). The solar modes that have nodes at the bottom of the simulation domain closely match the simulation modes. The simulation $p_{1}$ mode corresponds to the solar $p_{16}$ mode and the simulation $p_{2}$ mode corresponds to the solar $p_{26}$ mode. The simulation $p_{3}$ mode lies above the acoustic cutoff frequency.

The actual mode energy decay rate of the simulation modes, which on average is equal to their excitation rate, is obtained by multiplying the energy of each mode by its decay rate $\Gamma=2 \pi \Delta \nu_{\mathrm{FWHM}}$, which is its radian line width and is obtained from the fit to the modes (fig 1).

The total mode energy is the sum of the mode energies minus a fit to the background over the frequency range where the modes are significantly above the background multiplied by the area ( 36 $\mathrm{Mm}^{2}$ ) of the simulation domain (fig 1).

Eqn (5) is used to predict the mode excitation rate. The non-adiabatic total (gas + turbulent) pressure fluctuations are calculated directly from the simulation by first averaging the gas pressure, turbulent pressure and density over horizontal planes at each saved snapshot. These are then interpolated to the Lagrangian frame at each time. The non-adiabatic pressure fluctuations are calculated as in eqns A1 and A2. The oscillation modes of the domain are essentially adiabatic and do not affect the non-adiabatic pressure fluctuations as can be seen from its featureless, noisy spectrum 


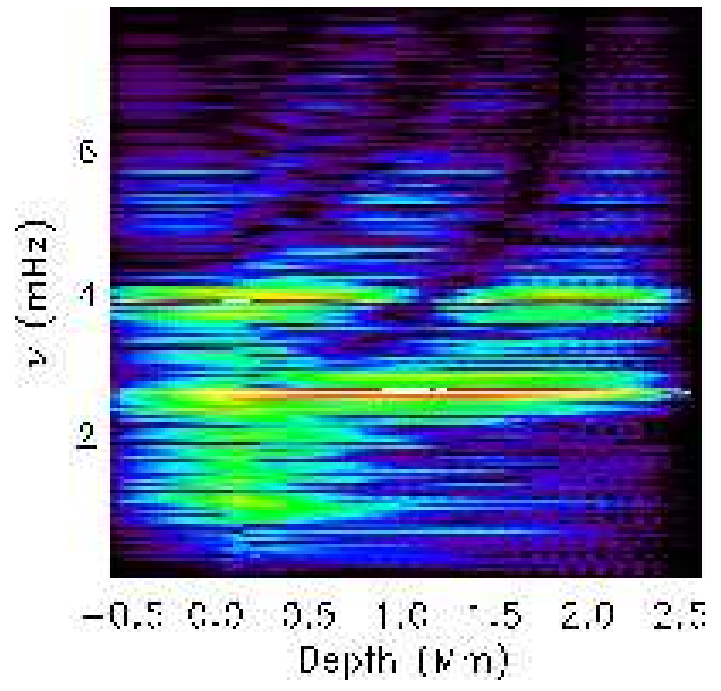

Fig. 3.- Logarithm of kinetic energy as a function of frequency and depth. The three resonant modes of the simulation stand out as maxima in the kinetic energy. A regular, continuous pattern of nodes and antinodes in the kinetic energy exists both at the resonant modes and between them.

(Fig 4). Thus the non-adiabatic pressure work is due primarily to the convection.

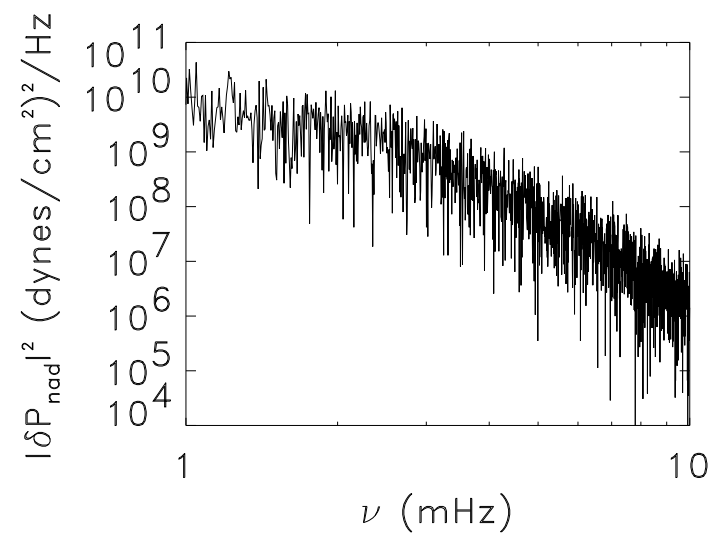

Fig. 4.- Non-adiabatic pressure spectrum at the a depth of $100 \mathrm{~km}$. Note that it is featureless even at the frequencies of the simulation modes. Hence, the non-adiabatic pressure is primarily due to random convective processes.

The mode compression is calculated from the Christensen-Dalsgaard modes (since these are es-

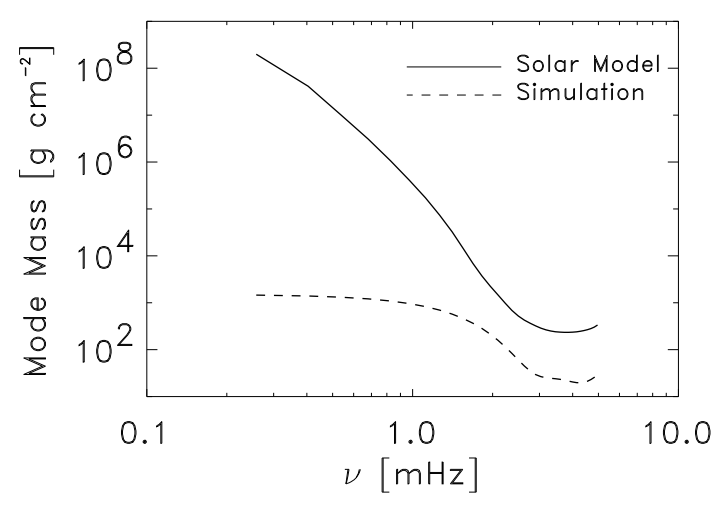

Fig. 5.- Mode mass in the simulation domain and the Sun. Mode mass decreases with increasing frequency because higher frequency modes are more concentrated toward the surface than low frequency modes. Because the solar modes extend below the bottom of the simulation domain, they have a larger mode mass. Because the simulation domain is shallow, the mode mass becomes nearly constant at low frequency where the eigenfunctions become nearly constant within the depth of the simulation.

sentially identical with the simulation modes at the resonant frequencies) normalized by the square root of their energy in the simulation domain. Because the simulation domain is shallow, while, especially at low frequency, the solar modes have substantial amplitude below the computational domain, the mode mass in the computational box is significantly smaller than the actual mode mass of the solar modes (Fig 5).

Finally, we compare the actual mode energy decay rate with the predicted rate of work by convection on the modes given by eqn (5) in figure 6 . The squares are the actual mode energy decay rates for the three resonant modes of the box. The solid line is the running mean of the predicted work and the dashed line is a smooth two power law fit to the predicted work. The agreement is very good, but not perfect. There are significant deviations from the power law fit in the neighborhood of the modes and these are reflected in the actual mode decay rate. This is a phenomena that still remains to be explained. Notice also that the decrease in work toward low frequencies is much slower than for the 


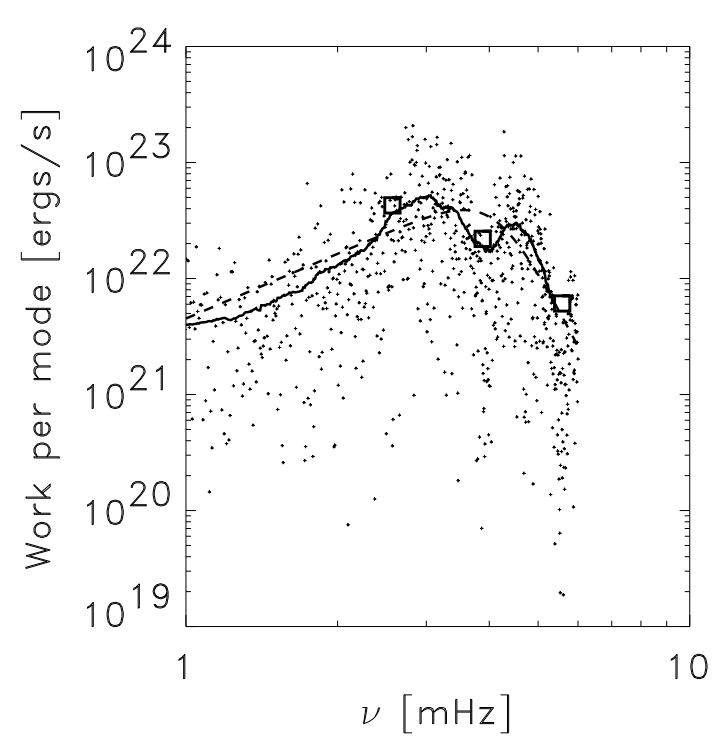

Fig. 6.- Rate of stochastic energy input to the simulation modes (squares) compared to the predicted excitation rate (small crosses) from the work of non-adiabatic pressure fluctuations on the modes (eqn 5). The solid line is a running boxcar average and the dashed line a two power law fit over the entire frequency range. The excitation rate is larger than the solar case because the mass of the modes in the simulation is less than the mass of the modes in the Sun. The near constancy of simulation mode mass at low frequency leads to a much slower decrease of excitation with decreasing frequency than occurs in the Sun.

Sun (Fig 7). The reason is the near constancy of the mode mass within the simulation domain at these low frequencies compared with the steeply rising mode mass with decreasing frequency on the Sun. This application of our excitation rate formula to the modes that are excited in our simulation verifies that the formula is correct and can be applied to the Sun.

\section{Mode Excitation: Sun}

\subsection{Excitation Spectrum}

The excitation rate for solar modes as a function of frequency is shown in Fig. 7. To obtain these results we used the shorter but higher resolution $125^{2} \times 82$ simulation because it has more

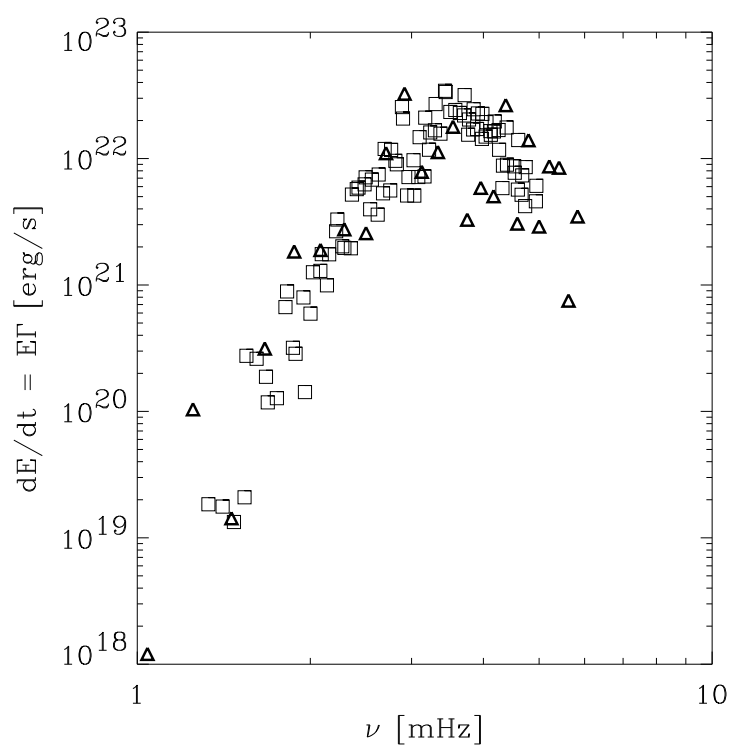

Fig. 7.- Rate of stochastic energy input to modes for the entire solar surface (simulation=triangles, observations=squares) from Roca Cortes (1999), based on observed mode velocity amplitudes and line widths from GOLF, for modes with $\ell=0-3$, which are all nearly radial close to the surface. The rate of energy input to the solar modes is smaller than to the simulation modes by the ratio of the mode mass of the solar modes to the mode mass in the simulation domain (fig 5).

high frequency power. The magnitude and frequency dependence we find for our $6 \mathrm{Mm}$ square box is very close to the observed values for the entire sun. This means that the pressure fluctuations must be uncorrelated on larger scales, so there is no extra driving contribution.

What produces this frequency dependence? The low frequency behavior is controlled by the nature of the eigenmodes and the high frequency behavior is controlled by the nature of convection. The work integral (Eqn. 5) contains a factor pertaining to the modes: the radial gradient of the mode displacement normalized by the square root of the mode energy (which makes it independent of the mode amplitude). This is small at low frequencies and increases with frequency approximately as $\nu^{3.5}$ (Fig 8). Part of this dependence is due to the radial gradient of the displacement. The mode dispersion relation is $\omega=\sqrt{ }(g k)$, so $k=\omega^{2} / g$, 


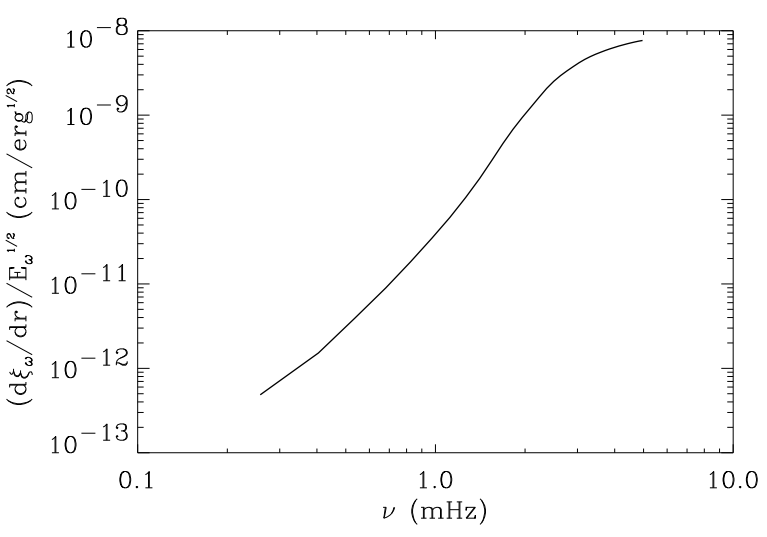

Fig. 8.- The mode factor in the work integral: $\frac{\partial \xi_{\omega}}{\partial z} / E_{\omega}^{1 / 2}$. Excitation decreases at low frequency due to mode behavior, in part because the radial wave vector is approximately $k=\omega^{2} / g$ and in part because the mode mass increases with decreasing frequency (Fig 5).

which accounts for two powers of the frequency. The remainder of the frequency dependence is due to the mode mass, which decreases with increasing frequency because higher frequency modes are more concentrated toward the surface (Fig. 5).

The mode excitation decreases at high frequency because the pressure fluctuation power decreases with increasing frequency roughly as $\nu^{-4}$ (Fig. 9). Convective motions, whose power decreases at small scales and high frequencies, produce the gas and turbulent pressure fluctuations. This then leads to a similar high frequency decline in the pressure spectrum.

Total pressure fluctuations are small at low frequency because the atmosphere is nearly in hydrostatic equilibrium. However, the turbulent pressure fluctuations are largest at low frequency because they are a convective effect and convection is a longer time scale phenomena. Hence, the gas pressure fluctuations must also be large and out of phase with the turbulent pressure fluctuations at low frequency in order to produce small total pressure fluctuations (Fig. 10). The turbulent pressure is small compared to the gas pressure $(\sim 15 \%)$, but the magnitude of fluctuations are comparable for the gas and turbulent pressures, so they can indeed cancel each other.

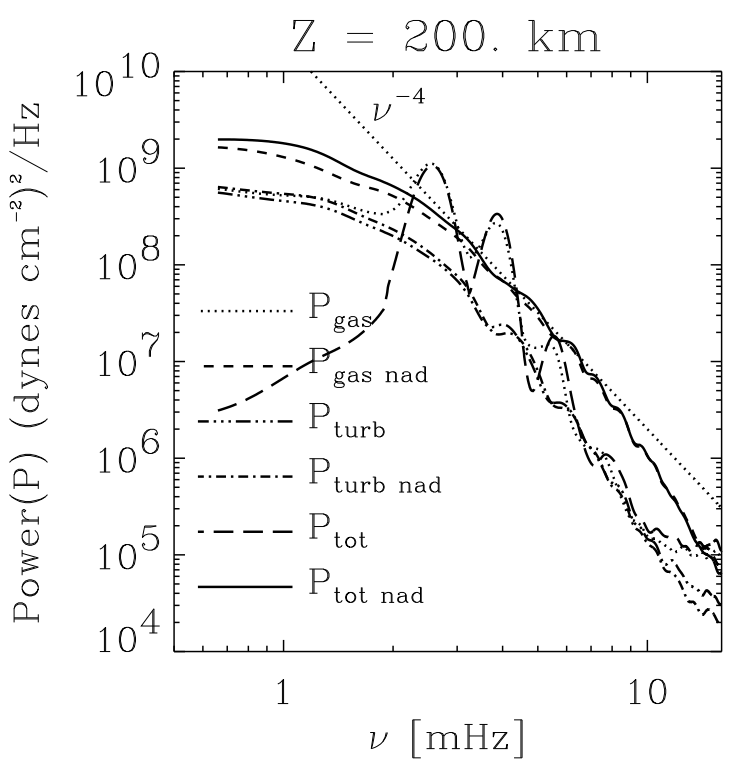

Fig. 9.- Spectrum of pressure fluctuations at a depth of $200 \mathrm{~km}$, smoothed with running boxcar mean. The non-adiabatic gas pressure fluctuations exceed the non-adiabatic turbulent pressure fluctuations by about a factor of four, but they become comparable in the peak driving range at larger depths. The local maxima in the total pressure fluctuations at 2.6, 3.9 and $5.5 \mathrm{mHz}$ are due to the resonant modes in the simulation. The non-adiabatic pressure varies smoothly across these resonant frequencies, indicating it is primarily due to convection. The pressure fluctuation power decreases roughly as $\nu^{-4}$ at high frequency, because it is due due to stochastic convective motions which decrease in power at high frequency.

\subsection{Excitation Location}

At what depth does the driving occur? Consider the integrand of the work integral at different frequencies (Figs. 11 and 12). At low frequencies the integrand amplitude is similar over an extended depth range, but it is small. Where the integrand is large, in the region of peak driving around $3-4 \mathrm{mHz}$, the integrand becomes concentrated very close to the surface and most driving occurs between the surface and $500 \mathrm{~km}$ depth. At still higher frequencies the integrand becomes small again and even more concentrated near the 


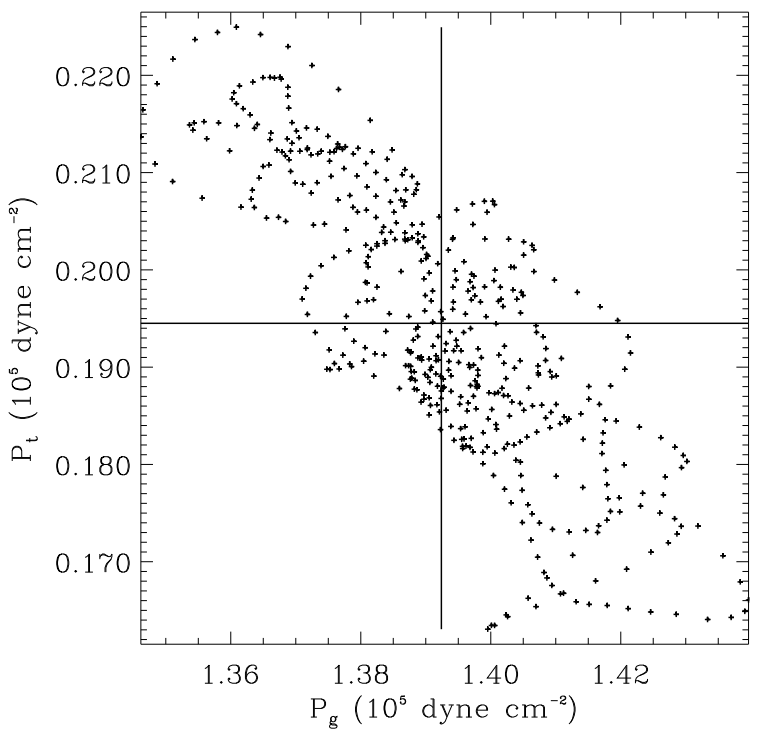

Fig. 10.- Correlation of turbulent and gas pressure at the surface. The turbulent pressure is only about $1 / 6$ the magnitude of the gas pressure near the surface but the magnitude of their fluctuations are similar.

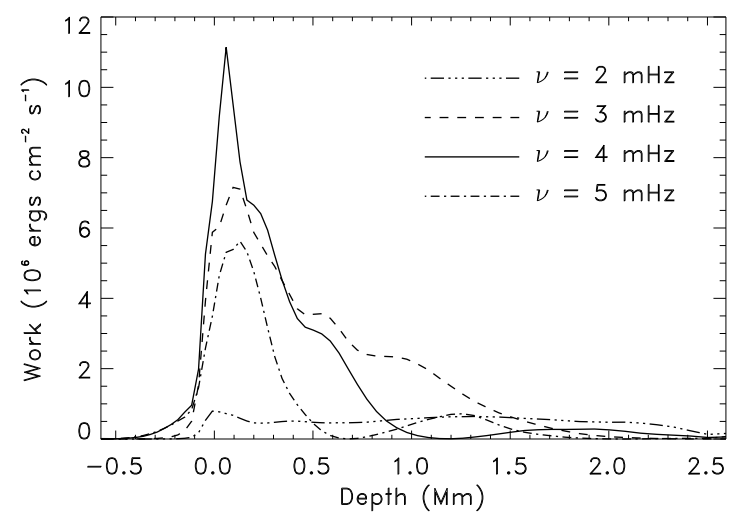

Fig. 11.- Integrand of the work integral, $\omega^{2}\left|\delta P_{\omega}^{*} \frac{\partial \xi_{\omega}}{\partial z}\right|^{2} / 8 \Delta \nu E_{\omega},($ eqn 5$)$ at $2,3,4$, and 5 $\mathrm{mHz}$ as a function of depth. At frequencies where the driving is large, the integrand is significant only within $500 \mathrm{~km}$ of the surface.

surface.

Where in space does this driving occur? The warm granules have only small non-adiabatic pres-

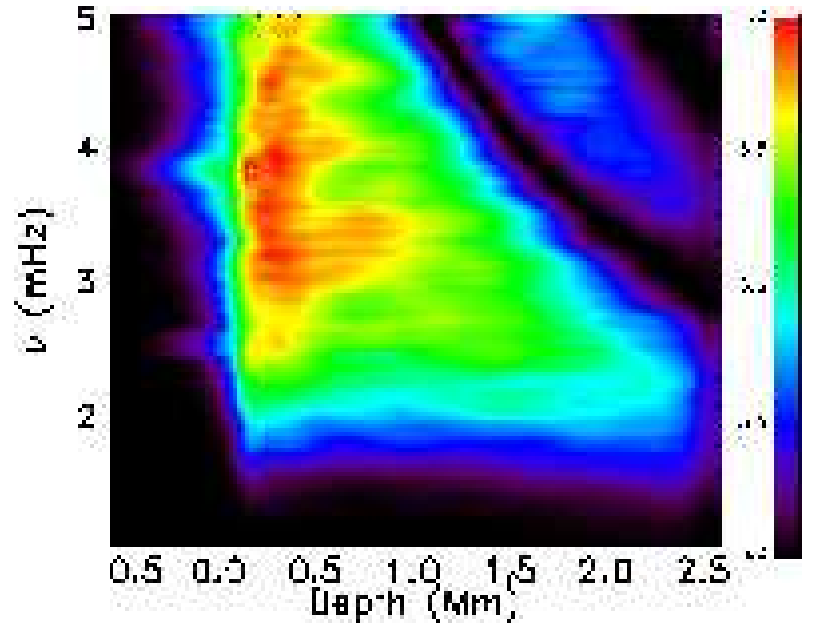

Fig. 12.- Logarithm (base 10) of the work integrand, $\omega^{2}\left|\delta P_{\omega}^{*} \frac{\partial \xi_{\omega}}{\partial z}\right|^{2} / 8 \Delta \nu E_{\omega}$, (eqn 5) (in units of $\mathrm{ergs} / \mathrm{cm}^{2} / \mathrm{s}$ ) as a function of depth and freqency. The work is concentrated close to the surface in the peak excitation range $(3-4 \mathrm{mHz})$ and at higher frequencies.

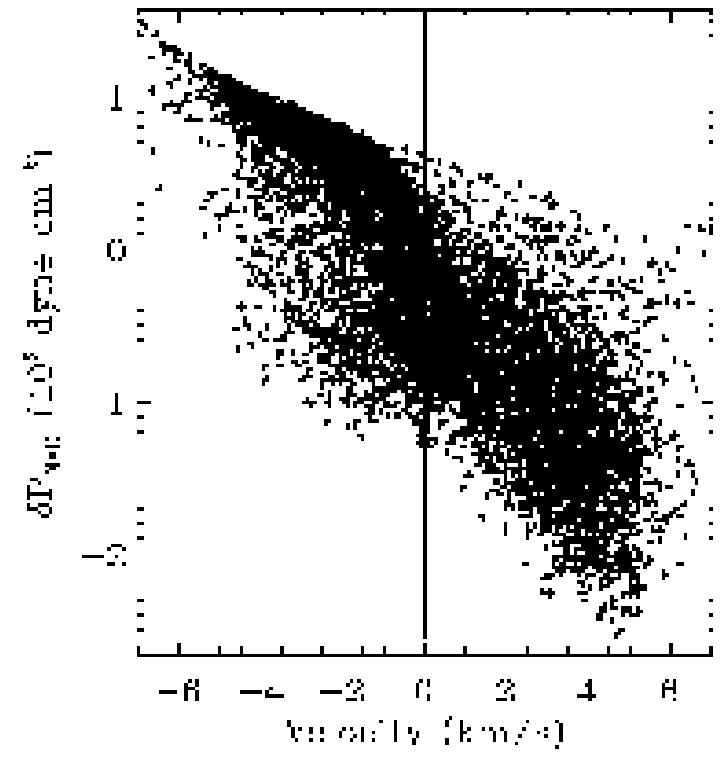

Fig. 13.- Correlation of non-adiabatic pressure with vertical velocity at the surface. The large negative fluctuations in the non-adiabatic pressure occur where the velocity is positive (downward) in the intergranular lanes.

sure fluctuations. Large, negative fluctuations are 


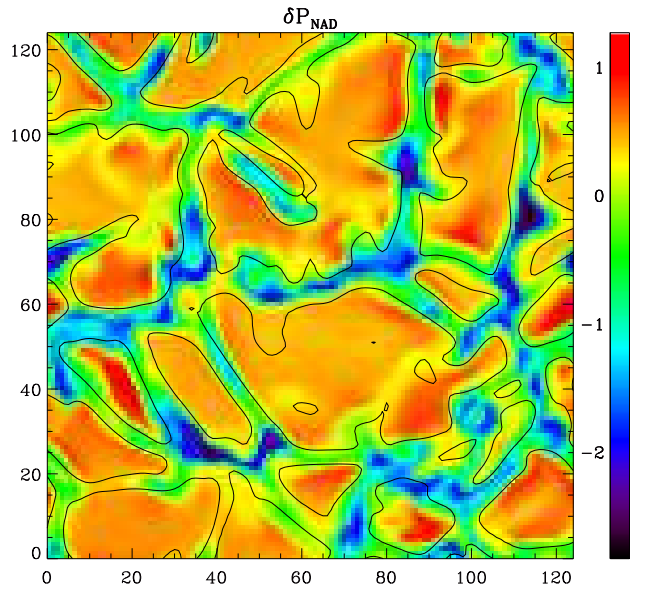

Fig. 14. - Image of the non-adiabatic total pressure fluctuations at depth $100 \mathrm{~km}$, with the contours of zero surface velocity to outline the granules. The units of the pressure are $10^{3}$ dynes $/ \mathrm{cm}^{2}$. Large, negative pressure fluctuations occur in the intergranular lanes. Positive pressure fluctuations are half as large and occur inside the granules.

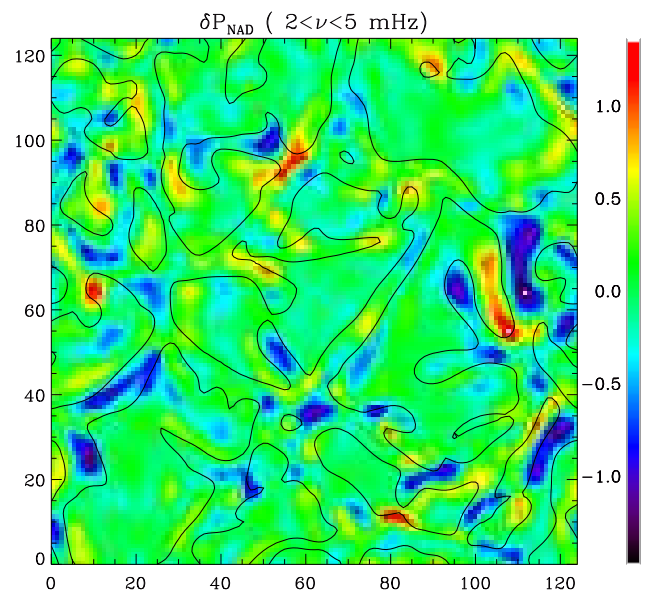

Fig. 15.- Image of non-adiabatic pressure fluctuations at $100 \mathrm{~km}$ depth in the $2-5 \mathrm{mHz}$ range, with the contours of zero velocity at the surface to outline the granules. The units of the pressure fluctuations are $10^{3}$ dyne $\mathrm{cm}^{-2}$. In this frequency range, where the driving is maximal, the largest $\delta P_{\text {nad }}$ occur at the edges of granules and inside the intergranular lanes.

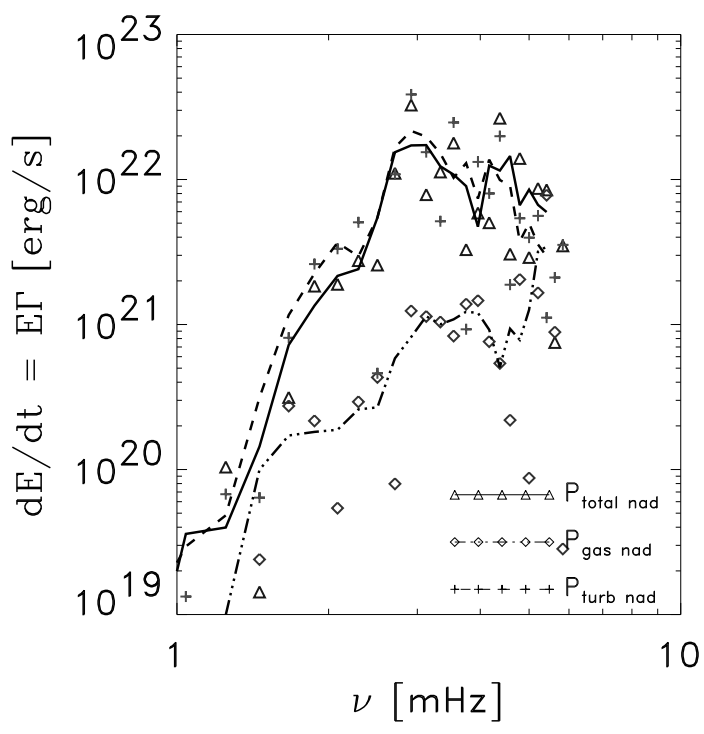

Fig. 16. - Rate of stochastic energy input to modes for the entire solar surface, showing the individual contributions of the non-adiabatic gas and turbulent pressure to the work of the total non-adiabatic pressure. Most of the driving in the peak driving range comes from the turbulent pressure.

concentrated in the downdrafts (Figs. 13, 14).

The maximum mode driving occurs in the frequency range of $3-4 \mathrm{mHz}$, by non-adiabatic pressure fluctuations in the same frequency range. By filtering the time sequence of these fluctuations we see (Fig 15) that in the peak driving range also the driving occurs predominantly in the intergranule lanes and near the edges of granules. The high frequency power near granule edges is due to the motion of the granule boundaries over the one hour time interval on which the filtering was performed. This is, in part, a result of changes in granule size as they evolve. No direct correlation of non-adiabatic pressure fluctuations in the range of $2-5 \mathrm{mHz}$ with velocity is seen. Keep in mind, however, that a correlation plot does not reveal correlations of events that happen in the neighborhood of one another. 


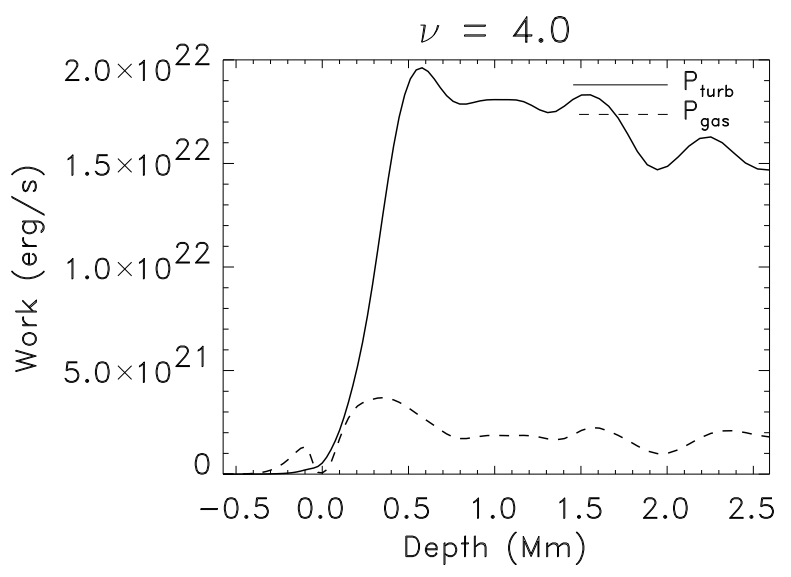

Fig. 17.- Mode driving at $4 \mathrm{mHz}$ evaluated from the surface to depth $\mathrm{z}$, showing the individual contributions of the non-adiabatic gas and turbulent pressure. Close to the surface the contributions of the two are comparable, but there is little contribution from the gas pressure below $200 \mathrm{~km}$ depth, while the turbulent pressure work is significant down to $500 \mathrm{~km}$ depth.

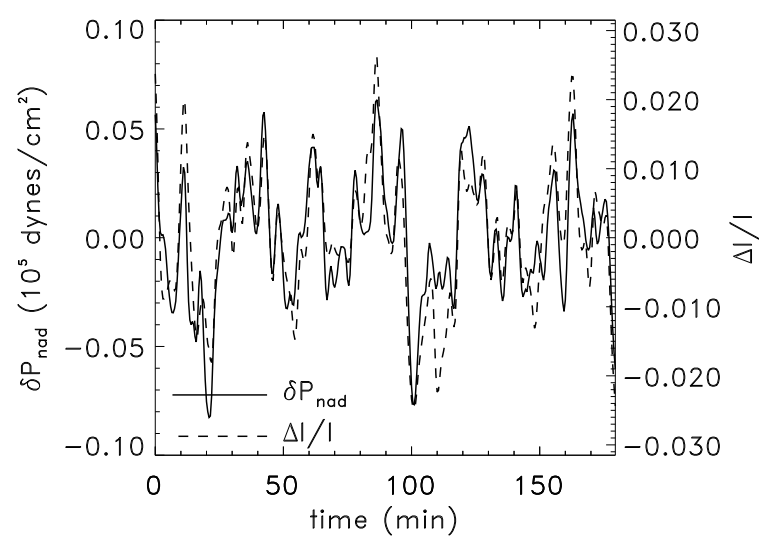

Fig. 18. - Horizontally averaged non-adiabatic pressure at the surface and emergent intensity variation in time. They are tightly correlated indicating that radiative cooling at the surface is the source of the non-adiabatic pressure fluctuations there..

\subsection{Excitation Source}

What is the source of the non-adiabatic pressure fluctuations? Is it entropy fluctuations or

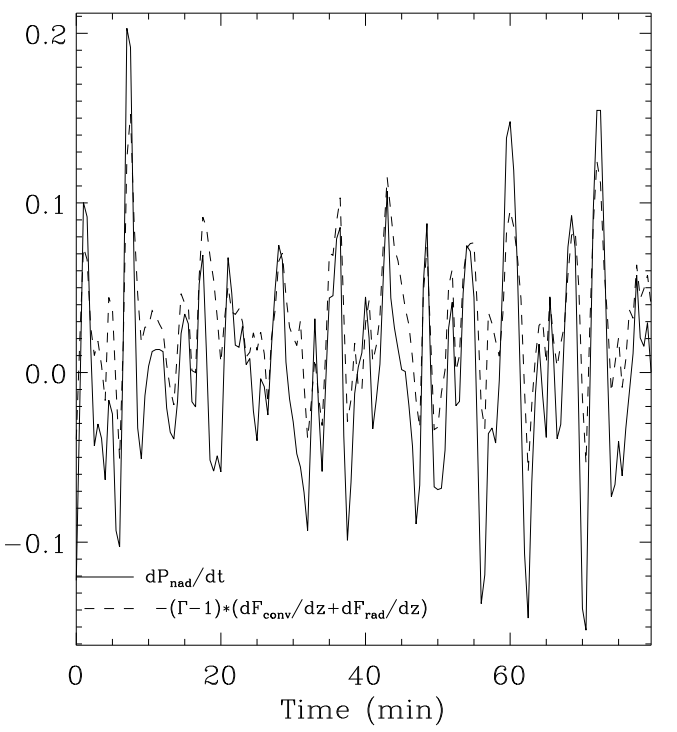

Fig. 19. - Divergence of the convective and radiative fluxes, at $\mathrm{z}=100 \mathrm{~km}$, multiplied by $\left(\Gamma_{3}-\right.$ 1) compared to the time derivative of the nonadiabatic pressure at the surface. The units are $10^{3} \mathrm{ergs} / \mathrm{cm}^{3} / \mathrm{s}$. The rate of change of nonadiabatic pressure closely follows the divergence of the net flux but has a slightly larger amplitude.

Reynolds stresses? Both play a role, but the primary source of mode driving is turbulent pressure fluctuations (Reynolds stresses) (Fig 16). This is surprising, since the non-adiabatic gas pressure power is larger than the turbulent pressure power near the surface (Fig 9). The non-adiabatic gas and turbulent pressures contribute comparably to the work near the surface, but the contribution of the turbulent pressure extends deeper and provides the dominant contribution to the total work (Fig 17).

The gas pressure fluctuations are significantly larger than the turbulent pressure and have maxima at the frequencies of the three radial modes of the simulation. The non-adiabatic pressure fluctuation power, however, varies smoothly across these frequencies indicating that it is primarily due to stochastic convective processes (Fig 9).

There is a tight correlation between the nonadiabatic pressure fluctuations at the surface and the emergent intensity (Fig 18), which indicates 


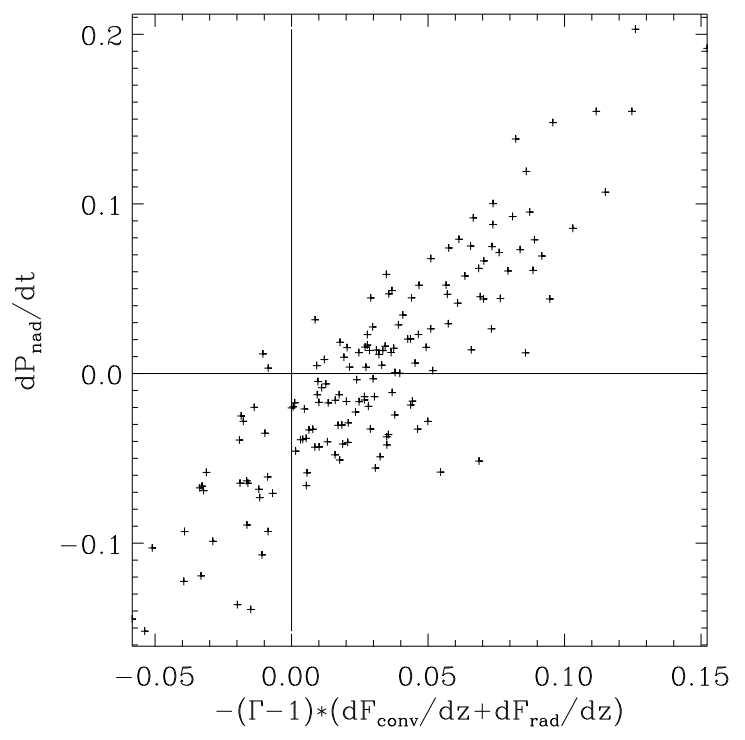

Fig. 20. - Correlation of the divergence of the convective and radiative fluxes, at $\mathrm{z}=100 \mathrm{~km}$, multiplied by $\left(\Gamma_{3}-1\right)$ with the time derivative of the non-adiabatic pressure at the surface. The units are $10^{3} \mathrm{ergs} / \mathrm{cm}^{3} / \mathrm{s}$. The close correlation shows that the slight instantaneous imbalance in the radiative and convective flux divergences is the primary source of non-adiabatic pressure fluctuations.

that it is the fluctuating cooling at the surface that is the main source of stochastic mode excitation there. Indeed, the source of entropy fluctuations is the cooling of fluid that approaches optical depth unity (Stein \& Nordlund 1998). This correlated noise is believed to be responsible for the difference in asymmetry of the modal power spectra observed in velocity and intensity (Nigam et al. 1998; Kumar \& Basu 1999). Our discussion in terms of non-adiabatic pressure fluctuations is equivalent to the discussion in terms of entropy fluctuations by Goldreich et al. (1994).

We can use the energy equation to determine the processes producing the non-adiabatic gas pressure fluctuations (Stein \& Nordlund 1991).

$\delta P_{\text {nad }}=\delta P-\frac{\Gamma_{1} P}{\rho} \delta \rho=\left(\frac{\partial P}{\partial s}\right)_{\rho} \delta s=\left(\Gamma_{3}-1\right) \delta Q$,

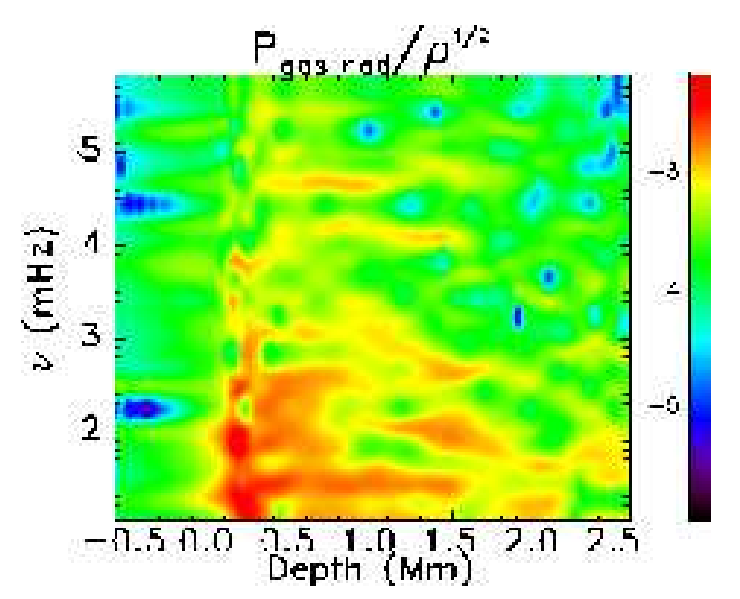

Fig. 21. - Logarithm of the gas pressure fluctuations scaled by the square root of the density, as a function of depth and frequency. The color scale is identical to the following image of the turbulent pressure fluctuations. With increasing frequency the gas pressure fluctuations become more rapidly concentrated near the surface and decrease more rapidly in strength than the turbulent pressure fluctuations.

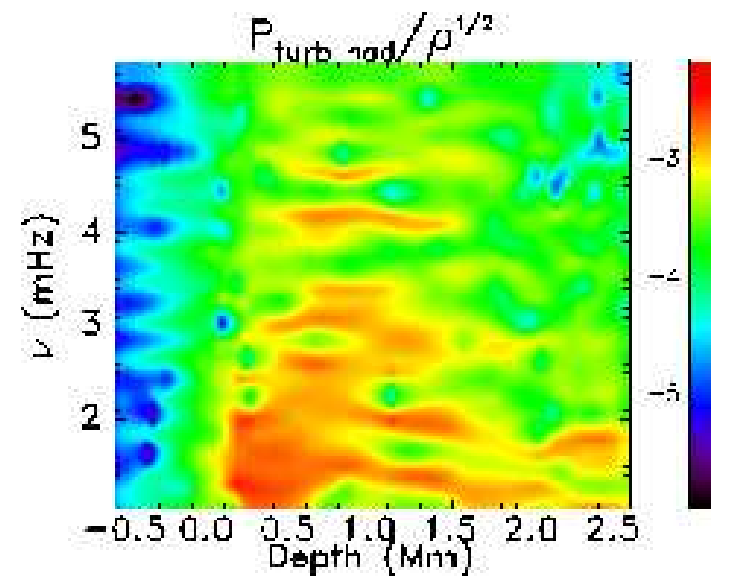

Fig. 22.- Logarithm of the turbulent pressure fluctuations scaled by the square root of the density, as a function of depth and frequency. The color scale is identical to the preceding image of the gas pressure fluctuations. The turbulent pressure fluctuations extend deeper and decrease less rapidly in magnitude with increasing frequency than the gas pressure fluctuations. As a result, turbulent pressure is the primary source of $p$-mode excitation in the peak driving range of $3-4 \mathrm{mHz}$. 


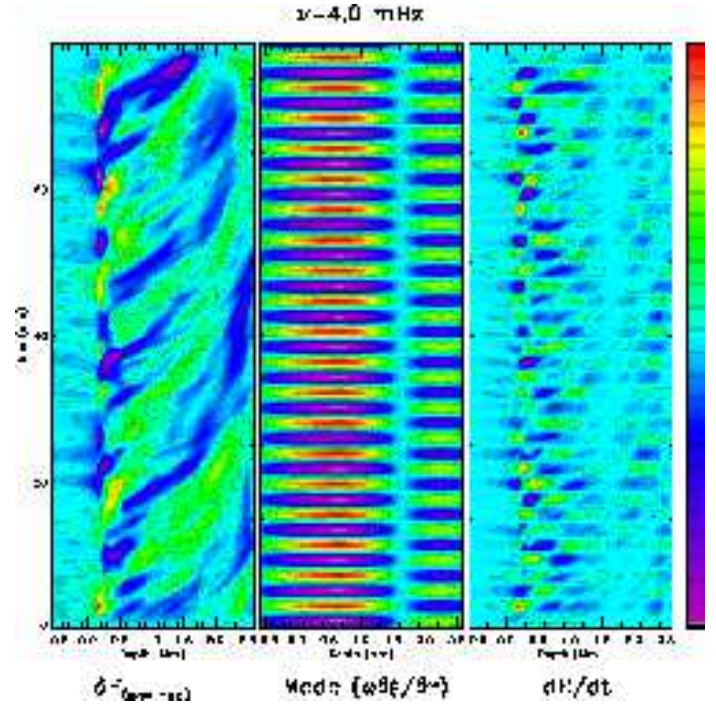

Fig. 23.- Integrand of the stochastic work integral for non-adiabatic gas pressure only, at $4 \mathrm{mHz}$, as a function of depth and time (right panel, eqn 5) and the two terms that contribute to it: the horizontally averaged, non-adiabatic gas pressure fluctuations, multiplied by $\langle\rho\rangle^{-1 / 2}$ (left panel) and the coherent mode density fluctuations multiplied by $\langle\rho\rangle^{1 / 2}$ (center panel). The non-adiabatic gas pressure fluctuations (left panel) are produced by the imbalance of the convective and radiative energy transport and are largest close to the surface. The color scale goes from maximum negative to maximum positive value for each variable.

and

$$
\frac{D Q}{D t}=-\frac{\partial}{\partial z}\left(F_{\text {rad }}+F_{\text {conv }}\right)+\breve{\mathbf{u}} \cdot \nabla P+Q_{\text {diss }},
$$

where $\breve{\mathbf{u}}=\mathbf{u}-\langle\rho \mathbf{u}\rangle /\langle\rho\rangle$ is the convective component of the velocity. The divergence of the radiative and convective fluxes are the dominant terms, but they nearly cancel each other since energy transport shifts from convective to radiative near the surface. It is their slight instantaneous imbalance locally that leads to the non-adiabatic pressure fluctuations (Figs 19 and 20).

The separate contributions of the gas and turbulent pressure to the total non-adiabatic pressure spectrum are shown in figs. 21 and 22, with the same color scale in both. Near the surface, there is clearly more power in the gas pressure. However, in the peak driving range of $3-4 \mathrm{mHz}$, there

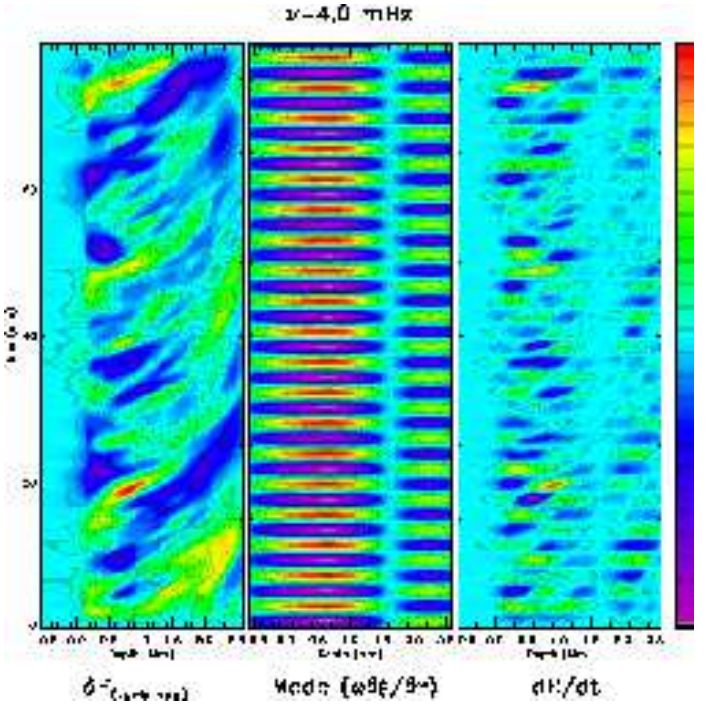

Fig. 24.- The same as figure 23, but for the nonadiabatic turbulent pressure only. The turbulent pressure fluctuations are more coherent in depth and time than the non-adiabatic gas pressure fluctuations so they produce a more coherent contribution to the work integral.

is more power in the non-adiabatic turbulent pressure below the surface.

Another reason for the dominance of turbulent pressure in the mode driving is revealed in figs. 23 and 24 . The left panels in each show the non-adiabatic gas and turbulent pressures (scaled by $\rho^{-1 / 2}$ ) as a function of time and depth. The turbulent pressure varies more slowly with depth and has a longer time scale than the gas pressure. The middle panels show one realization of the mode density or compression (scaled by $\left.\rho^{1 / 2}\right)$. The right panels show the work integrand, $\delta P_{\text {nad } \omega} \partial \xi_{\omega} / \partial z E_{\omega}^{-1 / 2}$. The gas pressure contribution to the integrand is more concentrated at the surface and alternates sign with time and depth more rapidly than the turbulent pressure contribution. The greater coherence of the turbulent pressure allows it to make a greater contribution to the net work.

The very largest pressure perturbations are associated with the sudden initiation of downdrafts and produce waves that propagate up into the chromosphere and steepen into shocks (Skartlien 1998; Skartlien et al. 2000). These events may cor- 


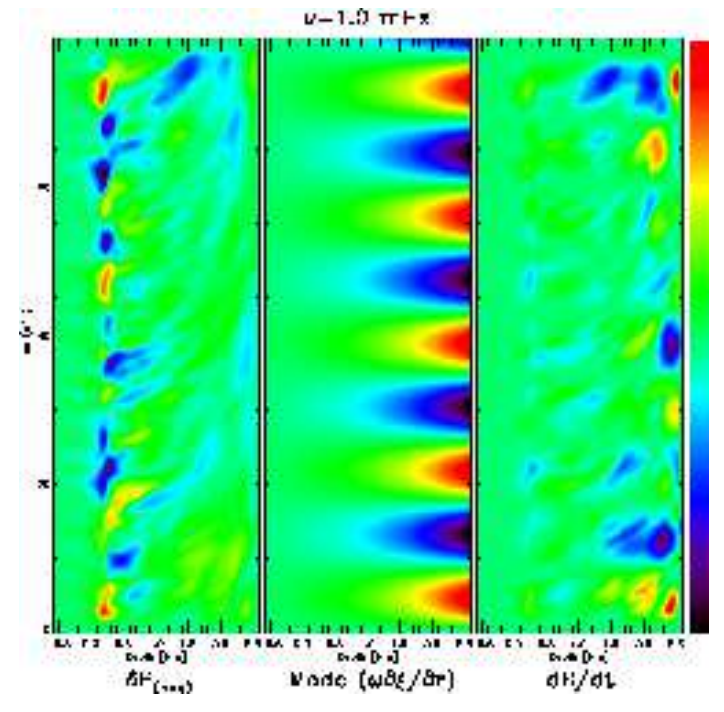

Fig. 25.- Integrand of the stochastic work integral as a function of depth and time (right panel, eqn 5) and the two terms that contribute to it: the horizontally averaged, non-adiabatic pressure fluctuations, multiplied by $\langle\rho\rangle^{-1 / 2}$ (left panel) and the coherent mode density fluctuations multiplied by $\langle\rho\rangle^{1 / 2}$ (center panel). The non-adiabatic pressure fluctuations (left panel) are produced by the imbalance of the convective and radiative energy transport and are largest close to the surface. The color scale goes from maximum negative to maximum positive value for each variable.

respond to the large individual acoustic events observed by Rimmele et al. (1995) and Goode et al. (1998). They may be the tail of the distribution of the stochastic driving process. A comparison of observed acoustic events and those in the simulation has been made by Goode et al. (1998).

\section{Summary}

How does this all fit together? Figs $25-27$ show the time variation of the horizontally averaged non-adiabatic pressure fluctuations, the density (compression) in the modes and the product of the two which is the integrand of the work integral (eqn 5) as a function of depth and time for modes with frequencies 1,3 , and $5 \mathrm{mHz}$.

The dominant contribution to the work comes from the turbulent pressure (Reynolds Stress). Near the surface non-adiabatic gas pressure fluc-

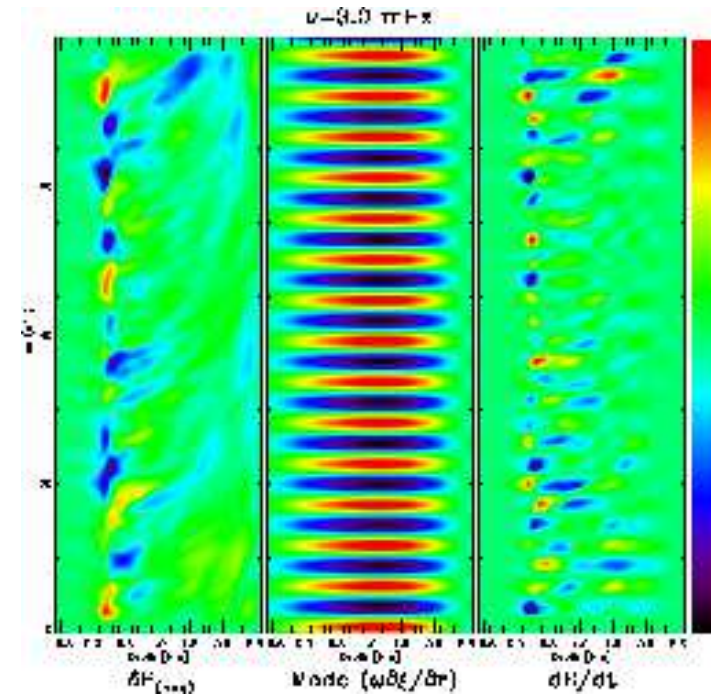

Fig. 26.- Same as Fig 25, but for $\nu=3 \mathrm{mHz}$. At higher frequencies the mode compression (center panel) and hence the work (right panel) becomes more concentrated toward the surface.

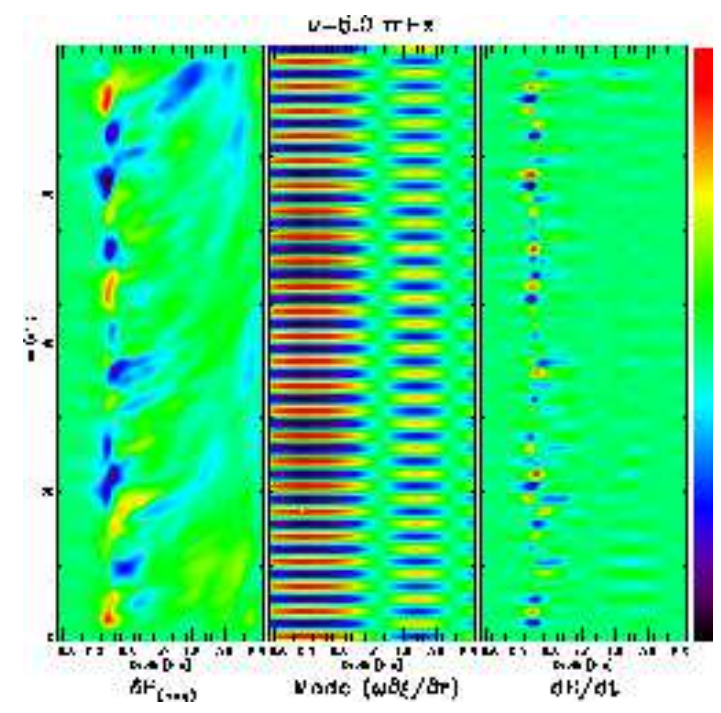

Fig. 27.- Same as Fig 25, but for $\nu=5 \mathrm{mHz}$. The compression and work are even more concentrated toward the surface and the mode has several radial nodes.

tuations, produced by an instantaneous imbalance between the divergences of the radiative and convective fluxes, also contribute. Their divergence is individually large, but since energy transport switches between convection and radiation in the 
surface layers, they are nearly equal and opposite. At each instant they do not exactly cancel, which leads to heating and cooling and hence entropy fluctuations. Excitation is small at low frequencies due to mode properties - the mode compression decreases and the mode mass increases at low frequency. In addition, at low frequency the mode amplitude is small near the surface where the nonadiabatic pressure fluctuations are large. (At very low frequencies, driving occurs deeper than the simulation domain.) Excitation is small at high frequencies due to the pressure fluctuation spectrum - pressure fluctuations become small at high frequencies because they are due to convective motions which have a longer time scale than the dominant $p$-mode periods. Large non-adiabatic pressure fluctuations occur primarily in the intergranular lanes (due to turbulence and surface radiative cooling) and near the edges of granules (due to granule expansion and collapse).

\section{Acknowledgments}

We thank Dali Georgobiani for making the 43 hour simulation. This work was supported in part by NASA grants NAG 5-4031 and NAG 58053, NSF grants AST 9521785 and AST 9819799, and the Danish Research Foundation, through its establishment of the Theoretical Astrophysics Center. The calculations were performed at the National Center for Supercomputer Applications, which is supported by the National Science Foundation, at Michigan State University and at $\mathrm{UNI} \bullet \mathrm{C}$, Denmark. This valuable support is greatly appreciated.

\section{REFERENCES}

Ando, H., Osaki, Y. 1977, PASJ, 29, 221

Asplund, M., Nordlund, A., Trampedach, R., Allende Prieto, C., Stein, R. F. 2000, Astronomy and Astrophysics (accepted), astro-ph/0005320

Balmforth, N. J. 1992, MNRAS, 255, 639

Biermann, L. 1948, Z. f. Astrophys., 25, 161

Bogdan, T. J., Cattaneo, F., Malagoli, A. 1993, ApJ, 407, 316

Christensen-Dalsgaard, J., Däppen, W., Ajukov, S. V., Anderson, E. R., Antia, H. M., Basu, S.,
Baturin, V. A., Berthomieu, G., Chaboyer, B., Chitre, S. M., Cox, A. N., Demarque, P., Donatowicz, J., Dziembowski, W. A., Gabriel, M., Gough, D. O., Guenther, D. B., Guzik, J. A., Harvey, J. W., Hill, F., Houdek, G., Iglesias, C. A., Kosovichev, A. G., Leibacher, J. W., Morel, P., Proffitt, C. R., Provost, J., Reiter, J., Jr., R., J., E., Rogers, F. J., Roxburgh, I. W., Thompson, M. J., Ulrich, R. K. 1996, Science, 272,1286

Crighton, D. G. 1975, Prog. Aerospace Sci., 16, 31

Goldreich, P., Keeley, D. A. 1977, ApJ, 212, 243

Goldreich, P., Kumar, P. 1990, ApJ, 363, 694

Goldreich, P., Murray, N., Kumar, P. 1994, ApJ, 424,466

Goode, P. R., Strous, L. H., Rimmele, T. R., Stebbins, R. T. 1998, apjl, 495, L27

Gustafsson, B., Bell, R., Eriksson, K., Nordlund, A. 1975, Astronomy and Astrophysics, 42, 407

Hyman, J. 1979, in R. Vichnevetsky, R. S. Stepleman (eds.), Adv. in Comp. Meth. for PDE'sIII, 313

Kumar, P., Basu, S. 1999, ApJ, 519, 389

Lighthill, M. J. 1952, Proc. R. Soc. London, A211, 564

Musielak, Z. E., Rosner, R., Stein, R. F., Ulmschneider, P. 1994, ApJ, 423, 474

Nigam, R., Kosovichev, A. G., Scherrer, P. H., Schou, J. 1998, ApJ, 495, L115

Nordlund, Å., Stein, R. F. 1990, Computer Phys. Communications, 59, 119

Nordlund, Å., Stein, R. F. 2000, ApJ (accepted), astro-ph/0006336

Rimmele, T. R., Goode, P. R., Harold, E., Stebbins, R. T. 1995, ApJ, 444, L119

Roca Cortes, T., Montanes, P., Palle, P. L., Perez Hernandez, F., Jimenez, A., Regula, C., the GOLF Team 1999, in A. Gimenez, E. Guinan, B. Montesinos (eds.), Theory and Tests of Convective Energy Transport, Vol. 173 of ASP Conf. Ser., 305 
Rosenthal, C. S., Christensen-Dalsgaard, J., Kosovichev, A. G., Nordlund, Å., Reiter, J., Rhodes, E. J., J., Schou, J., Stein, R. F., Trampedach, R. 1998, in SOHO 6/GONG 98: Structure and Dynamics of the Interior of the Sun and Sunlike Stars, ESA, p. (521

Rosenthal, C. S., Christensen-Dalsgaard, J., Nordlund, A.., Stein, R. F., Trampedach, R. 1999, A\&A, 351, 689

Schwarzschild, M. 1948, ApJ, 107, 1

Skartlien, R. 1998, 3D modeling of solar convection and atmosphere dynamics, $\mathrm{PhD}$ Thesis, University of Oslo

Skartlien, R., Stein, R., Nordlund, Å. 2000, ApJ (accepted)

Stein, R. F. 1967, Solar Physics, 2, 385

Stein, R. F. 1968, ApJ, 297, 154

Stein, R. F., Nordlund, Å. 1991, in D. Gough, J. Toomre (eds.), Challenges to Theories of the Structure of Moderate Mass Stars, Vol. 388 of Lecture Notes in Physics, Springer, Heidelberg, p. 195

Stein, R. F., Nordlund, Å. 1998, ApJ, 499, 914

Stein, R. F., Nordlund, Å. 2000, Solar Phys., (in press)

This 2-column preprint was prepared with the AAS LATEX macros v5.0. 


\section{A. Evaluation of the Mode Excitation Rate (eqn 5)}

The mode excitation rate (eqn 5) can be evaluated in two different ways: in Fourier space and in real space. We describe both methods. The variables that appear in eqn 5 for the mode excitation are the nonadiabatic total pressure fluctuation, the derivative of the mode displacement and the mode energy. These quantities are calculated as follows for both methods of evaluating the excitation.

The non-adiabatic pressure fluctuations are obtained from the simulation results. First the gas pressure, turbulent pressure $\left(P_{t}=\left\langle\rho u_{z}^{2}\right\rangle\right)$ and density are averaged over horizontal planes and saved at $10 \mathrm{~s}$ or 30 $\mathrm{s}$ intervals. These are then interpolated to the Lagrangian frame, that moves with the vertical (radial oscillation) motions. The Lagrangian frame is determined by calculating the mass column density at each time and interpolating the variables to the time average mass column density. Next, the non-adiabatic total (gas plus turbulent) pressure is calculated from

$$
P^{\text {nad }}=\left(\ln \left(P_{\text {gas }}+P_{\text {turb }}\right)-\Gamma_{1} \ln \rho\right)\left(P_{\text {gas }}+P_{\text {turb }}\right) .
$$

Finally, the fluctuation of the non-adiabatic total pressure about its time average,

$$
\delta P^{\text {nad }}(z, t)=P^{\text {nad }}(z, t)-\left\langle P^{\text {nad }}(z, t)\right\rangle_{t},
$$

is determined.

The mode displacement $\xi_{\nu}(z)$ for the radial mode of frequency $\nu$ is obtained from the eigenmode calculations of Christensen-Dalsgaard, using his spherically symmetric model S (Christensen-Dalsgaard et al. 1996). Alternatively, one could extract modes directly from the numerical simulations, using Fourier decomposition. A drawback with this method is, apart from slightly noisier mode structure, that there are only three resonant modes in the shallow simulation box - the 35 radial modes from standard solar models provide much better frequency coverage. As we have shown (fig 2), the modes from the simulation are essentially the same as the solar modes at the resonant frequencies of the computational domain, where the solar modes have a node at the depth of the bottom of the computational domain.

The mode energy is calculated from the displacement according to eqn. 6 . The mode displacements are interpolated to the simulation grid at the same total pressure as in the Christensen-Dalsgaard model and the derivative of the displacement is calculated. Since the derivative of the mode displacement always appears normalized by the square root of the mode energy, the mode amplitude normalization cancels.

In Fourier space, the work integral which appears in the energy input rate to the modes (eqn 5) is evaluated by taking the time Fourier transform of the total non-adiabatic pressure fluctuations at each depth, $\delta P^{\text {nad }}(z, t)$. For each frequency and depth, this is multiplied by the spatial derivative of the mode displacement at that depth interpolated to the frequencies of the Fourier transform (and normalized by the square root of the mode energy), $\omega\left(\partial \xi_{\omega} / \partial r\right) E_{\omega}^{-1 / 2}$. The spatial dependence of the modes varies slowly and continuously with frequency, so such interpolation is possible. This product is integrated over the depth of the simulation domain for each frequency. The energy input rate to the modes is the square of the absolute value of this work integral, divided by the frequency interval for the Fourier transform, $\Delta \nu$, (which equals multiplying by the time interval of the simulation), multiplied by the area of the simulation $\left(36 \mathrm{Mm}^{2}\right)$ and divided by eight (Eqn 5).

In real space, the integrand of the work integral for each frequency, $\nu$, is calculated as the non-adiabatic total pressure fluctuation at each depth and time, $\delta P^{\text {nad }}(z, t)$, multiplied by the normalized derivative of the mode displacement for that frequency at each depth, $\omega\left(\partial \xi_{\omega}(z) / \partial z\right) / E_{\omega}^{1 / 2}$, multiplied by a phase function $\cos (\phi+\omega t)$ for each time, $t$, a snapshot was saved in the simulation (10 s or $30 \mathrm{~s}$ intervals). For each depth, this is summed over all saved snapshots in the longest interval that is an integral number of mode periods for the given mode frequency $\nu$, and divided by the number of snapshots summed over to get the average value. This integrand is then integrated over depth. This is done for two values of the phase, $\phi=0$ and $\pi / 2$ (since the phases between the modes and the pressure fluctuations are random, we average these two orthogonal cases). The value of the integral for each phase is squared and these two values are summed. The energy input rate to the mode at this frequency is this average multiplied by the time interval integrated over (for that frequency), multiplied by the area of the simulation $\left(36 \mathrm{Mm}^{2}\right)$, and divided by eight. 